\title{
Triglycerides, apo C3 and Lp B:C3 and cardiovascular risk in Type II diabetes
}

\author{
N. Gervaise ${ }^{1}$, M. A. Garrigue ${ }^{2}$, G. Lasfargues ${ }^{1}$, P. Lecomte ${ }^{1}$ \\ ${ }^{1}$ Endocrine Unit, CHU Bretonneau, Tours, France \\ ${ }^{2}$ Biochemistry Laboratory, CHU Bretonneau, Tours, France
}

\section{Abstract}

Aims/hypothesis. Cardiovascular complications and particularly coronary heart disease are the main causes of morbidity and mortality in Type II (non-insulin-dependent) diabetes mellitus. Some studies have shown that hypertriglyceridaemia in diabetes is an independent cardiovascular risk factor. In the ECTIM study high apolipoprotein C3 and lipoprotein B:C3 concentrations (lipoparticles playing a role in triglyceride metabolism) were associated with myocardial infarction in non-diabetic subjects.

Methods. We studied the relations between macroangiopathy and different cardiovascular risk factors and lipid variables in 188 Type II diabetic subjects.

Results. Multivariate analysis showed that triglycer- ides, apo C3 and Lp B:C3, sex, duration of diabetes, microalbuminuria and age were independently associated with macroangiopathy. The study group was divided into quartiles according to apo C3 and Lp $\mathrm{B}: \mathrm{C} 3$ concentrations: the prevalence of macroangiopathy and coronary heart disease were increased in upper quartiles.

Conclusion/interpretation. Triglycerides apo C3 and Lp B:C3 were independent cardiovascular risk markers in our group of Type II diabetic patients. [Diabetologia (2000) 43: 703-708]

Keywords Type II diabetes, triglycerides, apolipoprotein C3, lipoprotein B:C3, cardiovascular risk, macroangiopathy, CHD.
Cardiovascular disease is a major cause of morbidity and mortality in Type II (non-insulin-dependent) diabetes mellitus. Most of the patients die from cardiovascular complications, particularly from ischaemic heart disease [1-3].

The mechanisms responsible for the increased cardiovascular risk in Type II diabetes are not fully understood. Diabetes is associated with many well-established cardiovascular risk factors. Patients with Type II diabetes have characteristic lipid abnormalities related to insulin resistance, i. e. hypertriglyceridaemia and low HDL-cholesterol [4-6]. The Paris Prospective Study [7] showed that hypertriglyceridaemia

Received: 12 August 1999 and in revised form: 7 January 2000

Corresponding author: N. Gervaise, Service de Médecine B, C.H.U. Bretonneau 37044 Tours Cedex, France

Abbreviations: Lp, Lipoprotein; apo, apolipoprotein; OR, odds ratio; TRLP, triglyceride-rich lipoprotein. is an independent cardiovascular risk factor among Type II diabetic patients.

A new lipoprotein classification was established more than 10 years ago by Alaupovic, Puchois and Fruchart, depending on apolipoprotein composition, which accounts for their functional specificity [8].

Among triglyceride-rich lipoproteins, the "Etude cas-témoin sur l'infarctus du myocarde" (ECTIM) Study $[9,10]$ emphasised the important role of Lp $\mathrm{B}: \mathrm{C} 3$ in the occurrence of myocardial infarction in non-diabetic patients.

There have been no studies reporting the relation between such lipoproteins and macrovascular disease or cardiovascular mortality in patients with Type II diabetes.

The purpose of this study was to assess relations between triglycerides, apo C3, Lp B:C3 and macroangiopathy, especially coronary heart disease in patients with Type II diabetes. 


\section{Subjects and methods}

Clinical characteristics. From September 1996 to December 1997, all patients with Type II diabetes attending the Endocrine Unit of the University Hospital in Tours, France, for a chronic complication or routine follow-up were entered in the study. Type II diabetic patients who were treated with diet alone, oral antidiabetic drugs or insulin or a combination of these were included. All Type I diabetic patients (low C-peptide or insulinaemia, ketoacidosis episodes) were excluded. Anthropometric data (height, weight) were collected for each patient and body mass index (BMI) was calculated. Information on tobacco smoking (patients were considered to be smokers if they had once smoked even if they were no longer smokers), age, duration of diabetes mellitus, the type of diabetic treatment (antidiabetic oral drugs: metformin, sulphonylureas, $\alpha$-glucosidase inhibitors, and/or insulin therapy), the existence and type of hypolipidaemic treatment (fibrates, statins, fish oils) were recorded. Subjects were considered to suffer from hypertension if blood pressure was above $140 / 80 \mathrm{mmHg}$ or if they were taking antihypertensive drugs.

Diabetic macroangiopathy was defined by the presence of coronary heart disease, peripheral vascular disease or cerebrovascular disease or more than one of these.

Coronary heart disease was diagnosed in patients with: (1) a history of myocardial infarction (defined by chest pain of at least $20^{\prime}$ with increase in cardiac enzymes and typical ECG changes during an acute attack) or angina pectoris (with evidence of electrocardiographic ischaemia on conventional resting ECG) or both and/or (2) positive effort test (if chest pain occurred and/or if it was associated with an ST depression greater than $1 \mathrm{~mm}$ ) and/or (3) positive myocardial thallium scintigraphy combined with dipyridamole test (defined as the presence of reversible or fixed perfusion defect) and/or (4) positive coronary angiography (defined as a $>50 \%$ stenosis in at least one major vessel).

Peripheral vascular disease, clinically assessed by intermittent claudication and/or decubitus cramping based on the World Health Organisation (WHO) criteria [11] and/or arterial ulcers was confirmed by the presence of a greater than $50 \%$ stenosis on ultrasound examination with Doppler (Spectradop, DMS, Montpellier, France) and/or arteriography of lower limbs.

Cerebrovascular disease was defined by a history of ischaemic stroke (documented history of transient ischaemic attack, reversible ischemic neurological deficit or stroke caused by cerebral infarction) and/or stenosis ( $>70 \%$ ) or obliteration of carotid arteries on Doppler examination and/or evocative image on a cranial tomogram.

Diabetic microangiopathy was diagnosed by the presence of: (1) retinopathy, confirmed by fluorescein angiography whether or not treated by laser, and/or (2) diabetic neuropathy assessed by clinical findings (hypopallaesthesia and/or abnormal monofilament findings) and/or electromyogram.

Microalbuminuria was considered independently from other microangiopathic complications because it is a cardiovascular risk factor by itself in Type II diabetic subjects [12].

All blood samples were collected after at least $12 \mathrm{~h}$ of fasting. Explorations of lipid variables included measurement of total and HDL-cholesterol, triglycerides, lipoprotein(a), apolipoproteins $\mathrm{A} 1, \mathrm{~B}, \mathrm{C} 3$ and lipoprotein $\mathrm{B}: \mathrm{C} 3$. We also measured $\mathrm{HbA}_{1 \mathrm{c}}$ (normal 3.9 to $5.7 \%$ ) and microalbuminuria in a 24-h urine sample. Renal insufficiency was defined by creatininaemia above $150 \mu \mathrm{mol} / \mathrm{l}$.

Biochemical analysis. Plasma cholesterol was measured by an automated [using Hitachi 911 (Tokyo, Japan) autoanalyser] enzymatic (cholesterol oxydase) and colorimetric method (Cholesterol CHOD-PAP, Roche Diagnostics, Meylan, France).

The same method was used for the measurement of HDLcholesterol after precipitation of chylomicrons and low density lipoproteins (LDL) and very low density lipoproteins (VLDL) (HDL cholesterol/phospholipides, Biomerieux, Lyon, France).

Triglycerides were measured with an enzymatic (glycerol phosphate oxidase) and colorimetric (PAP) method (triglyceride GPO-PAP, Roche Diagnostics), using the Hitachi 911 autoanalyser.

Apolipoproteins A1 and B were measured with a nephelemetric kinetic method (APA-APB, Beckman-Coulter Instruments, Villepinte, France) with an Array 360 automate (Beckman-Coulter Instruments, Villepinte, France) and Lp(a) by an ELISA method using a second antibody stained with peroxidase (Tint Elise, Lpa Biopool, Diamed, Paris, France).

For apo C3 and Lp B:C3, an electroimmunodiffusion technique was used with agarose gel containing anti-apo C3 antibodies (Hydragel Lp CIII, Sebia, Issy-les-Moulineaux, France). Total Lp C3 was measured after electrophoresis of native serum. We obtained Lp C3-non B after precipitation with anti-apo B antibodies and electroimmunodiffusion of the supernatant. Concentrations of Lp B:C3 were calculated according to the difference between Lp C3 and Lp C3-non B. The intra-assay variation coefficient with this method was $3 \%$ and the inter-assay variation coefficient was $5 \%$.

We measured microalbuminuria using a nephelemetric kinetic technique (Microalbumine, Beckman-Coulter Instruments).

Haemoglobin $\mathrm{A}_{1 \mathrm{c}}$ was measured by turbidimetry after formation of immune complexes with excess of anti-HbA $\mathrm{Ac}_{1 \mathrm{c}}$ antibodies and agglutination with polyhaptens (Tina-quant $\mathrm{HA}_{1 \mathrm{c}}$, Roche Diagnostics) with an automated method (Hitachi 911).

Concentrations of LDL-cholesterol were calculated using the Friedwald formula: LDL-c $(\mathrm{g} / \mathrm{l})=$ total cholesterol $(\mathrm{g} / \mathrm{l})$-triglycerides (g/l)/5-HDL-c (g/l), if triglycerides were below $4 \mathrm{~g} / \mathrm{l}$.

Statistical analysis. Results were expressed as means \pm SD and/ or median and extreme values whenever the variable distribution was not normal.

Statistical analyses were done with the BMDP statistical package (Solo for Windows from BMDP Statistical Software, Los Angeles, Calif., USA). Differences between means or proportions were tested using one-way analysis of variance (ANOVA) or the chi-squared test, respectively. A non-parametric test (Mann and Whitney U test) was used when conditions of ANOVA were not respected. Statistical significance was defined as a two-tailed $p$ value of 0.05 or less.

Associations between continuous variables were assessed with the Pearson correlation coefficient and between categorical variables by the Mantel-Hanszel chi-squared test for trend. The independent associations of risk factors and macroangiopathy or coronaropathy were assessed with multiple logistic regression. Odds ratio followed by confidence intervals (CI) were calculated with regression coefficients [13]. Regression logistic models were chosen to estimate the cut-off values giving the best sensitivity of different variables for the risk of macroangiopathy and CHD.

\section{Results}

We prospectively included 188 patients with Type II diabetes in this cross-sectional study, [98 men $(52.1 \%)$ and 90 women $(47.9 \%)]$. The mean age was 
Table 1. Prevalence of diabetic complications in Type II diabetic subjects

\begin{tabular}{lr}
\hline Variable & $n(\%)$ \\
\hline Macroangiopathy & $81(32.3)$ \\
Coronary heart disease & $38(20.2)$ \\
Peripheral vascular disease & $39(20.7)$ \\
Cerebrovascular disease & $23(12.5)$ \\
Microangiopathy (retinopathy and/or neuropathy) & $55(29.3)$ \\
Microalbuminuria & $60(31.9)$ \\
Hypertension & $100(53.1)$ \\
\hline
\end{tabular}

Table 2. Lipid, lipoprotein and apolipoprotein concentrations (results are expressed as means \pm SD and as medians with the extreme values)

\begin{tabular}{lcc}
\hline & Means \pm SD & Median (extremes) \\
\hline Total cholesterol $(\mathrm{mmol} / \mathrm{l})$ & $5.58 \pm 1.37$ & $5.54(2.53-11.6)$ \\
Triglycerides $(\mathrm{mmol} / \mathrm{l})$ & $2.14 \pm 1.73$ & $1.67(0.51-16.5)$ \\
HDL-cholesterol $(\mathrm{mmol} / \mathrm{l})$ & $1.22 \pm 0.44$ & $1.10(0.29-3.67)$ \\
LDL-cholesterol $(\mathrm{mmol} / \mathrm{l})$ & $3.41 \pm 1.13$ & $? ? ? ?$ \\
Lipoprotein $(\mathrm{a})(\mathrm{g} / \mathrm{l})$ & $0.235 \pm 0.292$ & $0.129(0.010-1.51)$ \\
Apo A1 $(\mathrm{g} / \mathrm{l})$ & $1.40 \pm 0.32$ & $1.37(0.53-2.74)$ \\
Apo B $(\mathrm{g} / \mathrm{l})$ & $1.28 \pm 0.40$ & $1.22(0.48-2.67)$ \\
Apo C3 $(\mathrm{mg} / \mathrm{l})$ & $34.86 \pm 16.94$ & $31.00(5-162)$ \\
Lp B:C3 $(\mathrm{mg} / \mathrm{l})$ & $18.38 \pm 12.95$ & $16.00(2-102)$ \\
\hline
\end{tabular}

59 years $( \pm 12.8)$. Diabetes had been diagnosed about 10 years before (mean $9.78 \pm 8.82$ years). There were $88(46 \%)$ active or former smokers. Mean BMI was $29.8( \pm 6.05) \mathrm{kg} / \mathrm{m}^{2}$. Of the patients $50(26.7 \%)$ were treated with insulin therapy, 124 (66\%) with antidiabetic oral drugs: 85 (45.5\%) with sulphonylureas, 97 (51.9\%) with metformin and 19 $(10.2 \%)$ with $\alpha$-glucosidase inhibitors. There were $29(15.4 \%)$ who were treated by diet alone and 36 (19\%) with hypolipaemic drugs: 15 (8\%) with HMG-CoA reductase inhibitors, 27 (14.4\%) with fibrates and $4(2 \%)$ with fish oils.

The prevalence of diabetic complications and hypertension is presented in Table 1 . Of the patients 14 suffered from chronic renal insufficiency. Mean $\mathrm{HbA}_{1 \mathrm{c}}$ was $8 \%( \pm 2.2)$. The lipid values are summarised in Table 2. Concentrations of LDL were not available for 14 subjects because their triglyceride concentrations were above $4 \mathrm{~g} / \mathrm{l}$. Triglycerides, apo $\mathrm{C} 3$ and Lp B:C3 were strongly correlated (triglycerides and apo C3: $r=0.80 ; p<0.001$ and triglycerides and Lp B:C3: $r=0.79 ; p<0.001)$.

Bivariate analysis. Macroangiopathy was significantly associated with age, duration of diabetes mellitus, sex (data not shown), creatinine concentration, microalbuminuria and microangiopathy. Among lipid variables, triglycerides, apo C3 and Lp B:C3 were significantly associated with macroangiopathy (Table 3 ).

Coronary heart disease was significantly associated with age, sex (not shown), microalbuminuria and microangiopathy. Concerning lipid variables, a significant association was observed for triglycerides, apo C3, Lp B:C3, and Lp(a) (Table 3).

Effects of treatments. Triglyceride, apo C3 and Lp B:C3 concentrations were statistically significantly lower in subjects treated with insulin. Their $\mathrm{HbA}_{1 \mathrm{c}}$ values were similar to those of subjects on diet alone or treated with oral antidiabetic treatment (data not shown). Of the subjects $19 \%$ were receiving hypolipidaemic treament. After exclusion of subjects treated with the above drugs, the statistic association between triglycerides, apo C3, Lp B:C3 and macroangiopathy or coronaropathy remained statistically significant (data not shown).

Multivariate analysis. All lipid variables were submitted to multivariate analysis using multiple logistic regression (Table 2). Age, duration of diabetes, BMI, $\mathrm{HbA}_{1 \mathrm{c}}$, sex, drugs, microalbuminuria and hypertension were also considered.

Logistic regression models taking into account either triglycerides, apo C3 or Lp B:C3 indicated an in-

Table 3. Associations with macroangiopathy and coronary heart disease in univariate analysis

\begin{tabular}{|c|c|c|c|c|c|c|}
\hline & Mac - & $\mathrm{Mac}+$ & $p$ & $\mathrm{CHD}-$ & $\mathrm{CHD}+$ & $p$ \\
\hline Age (years) & $57 \pm 11$ & $64 \pm 10$ & $<0.001$ & $58 \pm 11$ & $65 \pm 11$ & 0.001 \\
\hline Duration of diabetes (years) & $8 \pm 8$ & $12 \pm 9$ & $<0.001$ & $9 \pm 8$ & $12 \pm 8$ & 0.06 \\
\hline $\operatorname{BMI}\left(\mathrm{kg} / \mathrm{m}^{2}\right)$ & $30.2 \pm 6.2$ & $29.2 \pm 5.8$ & NS & $29.8 \pm 6$ & $30.1 \pm 6.1$ & NS \\
\hline Creatinine $(\mu \mathrm{mol} / \mathrm{l})$ & $85 \pm 18$ & $106 \pm 63$ & 0.001 & $90 \pm 33$ & $106 \pm 65$ & NS \\
\hline Total cholesterol (mmol/l) & $5.48 \pm 1.43$ & $5.73 \pm 1.26$ & NS & $5.48 \pm 1.42$ & $5.87 \pm 1.18$ & 0.08 \\
\hline Triglycerides (mmol/l) & $1.93 \pm 1.25$ & $2.50 \pm 2.30$ & 0.02 & $2.07 \pm 1.77$ & $2.39 \pm 1.64$ & 0.03 \\
\hline $\operatorname{Lp}(a)(g / l)$ & $0.22 \pm 0.29$ & $0.26 \pm 0.28$ & 0.06 & $0.22 \pm 0.29$ & $0.28 \pm 0.27$ & 0.04 \\
\hline Apo A1 (mg/l) & $1.42 \pm 0.34$ & $1.36 \pm 0.26$ & NS & $1.40 \pm 0.32$ & $1.39 \pm 0.29$ & NS \\
\hline Aро B (mg/l) & $1.26 \pm 0.41$ & $1.29 \pm 0.39$ & NS & $1.25 \pm 0.41$ & $1.34 \pm 0.39$ & NS \\
\hline Apo C3 (mg/l) & $32.4 \pm 13.3$ & $39.9 \pm 21.3$ & 0.007 & $33.9 \pm 17.9$ & $38.1 \pm 13.4$ & 0.006 \\
\hline Lp B:C3 (mg/l) & $16.7 \pm 11.3$ & $21.3 \pm 15.0$ & 0.007 & $17.7 \pm 13.6$ & $20.7 \pm 10.6$ & 0.01 \\
\hline
\end{tabular}

Mac -: subjects without macroangiopathy, Mac +: subjects with macroangiopathy; CHD -: subjects without coronary heart disease, $\mathrm{CHD}+$ : subjects with coronary heart disease, $p<0.05$ significant Means \pm SD 
Table 4. Variables independently associated with macroangiopathy in logistic regression models in Type II diabetic patients

\begin{tabular}{lll}
\hline Variables (reference) & Odds ratio (CI 95\%) & $p$ \\
\hline Lp B:C3 $(<17 \mathrm{mg} / \mathrm{l})$ & $2.73(1.33-5.60)$ & 0.005 \\
Microalbuminuria $(<30 \mathrm{mg} / 24 \mathrm{~h})$ & $2.45(1.20-4.99)$ & 0.01 \\
Sex (female) & $2.38(1.17-4.76)$ & 0.01 \\
Age $(<60$ years) & $2.28(1.10-4.73)$ & 0.02 \\
Duration of diabetes $(<10$ years) & $2.11(1.04-4.27)$ & 0.03 \\
\hline
\end{tabular}

Table 5. Comparison with apo C3 and Lp B:C3 concentrations of the French control group without diabetes in the ECTIM study

\begin{tabular}{lcc}
\hline & $\begin{array}{l}\text { ECTIM France } \\
(n=432)\end{array}$ & $\begin{array}{c}\text { Type II diabetic } \\
\text { subjects }(n=188)\end{array}$ \\
\hline Age $(\text { years })^{\mathrm{a}}$ & $53.3 \pm 8.6$ & $59 \pm 12.8$ \\
BMI $\left(\mathrm{kg} / \mathrm{m}^{2}\right)^{\mathrm{b}}$ & $26.6 \pm 3.8$ & $29.8 \pm 6.05$ \\
Hypolipidaemic drug $(\%)^{\mathrm{b}}$ & 6.3 & 19 \\
apo C3 $(\mathrm{mg} / \mathrm{l})^{\mathrm{c}}$ & $29.2 \pm 11.2$ & $34.86 \pm 16.94$ \\
$\mathrm{Lp} \mathrm{B:C3}(\mathrm{mg} / \mathrm{l})^{\mathrm{c}}$ & $9.3 \pm 5.9$ & $18.38 \pm 12.95$ \\
\hline${ }^{\mathrm{a}} p=\mathrm{NS},{ }^{\mathrm{b}} p<0.001,{ }^{\mathrm{c}} p<0.00001$ &
\end{tabular}

dependent association of each of these variables with macroangiopathy. The most significant odds ratio was that for Lp B:C3.

Five factors remained independently associated with macroangiopathy in the final model of multiple logistic regression (Table 4). The increased risk of macroangiopathy was related to $\mathrm{Lp} \mathrm{B}: \mathrm{C} 3$ above $17 \mathrm{mg} / \mathrm{l}$, microalbuminuria above $30 \mathrm{mg} / 24 \mathrm{~h}$, male sex, age above 60 years and duration of diabetes above 10 years. The highest odds ratio (OR) was obtained with Lp B:C3. For coronary heart disease, only Lp B:C3 (OR 3.95, CI 1.73-9.04) and sex (OR 2.32, CI 1.01-5.26) remained independently associated.

Relation between Lp B:C3 and macroangiopathy or $C H D$. The study group was divided into quartiles of Lp B:C3 (in quartile $1 \mathrm{LpB}: \mathrm{C} 3<10 \mathrm{mg} / \mathrm{l}$; in quartile $2, \geq 10$ to $<16$; in quartile $3, \geq 16$ to $<23$; in quartile $4, \geq 23)$. The prevalence of macroangiopathy increased with increasing quartiles. The odds ratio was calculated as the ratio of the prevalence in a given quartile of $\mathrm{Lp} \mathrm{B:C3}$ in relation to the prevalence in quartile 1 . Odds ratio for macroangiopathy were 2.06 in quartile $2,3.46$ in quartile 3 and 6.24 in quartile 4 (adjusted OR 1.63; $p<0.004$ ), and for CHD respectively 2.71, 4.98, 4.45 (adjusted OR 1.64; $p<0.006)$.

Comparison of apo $C 3$ and $L p$ B:C3 concentrations with the French cohort of the ECTIM Study (Table 5). The French control group of the ECTIM study was composed of 432 men, whose anthropometric data are summarised in Table 5. Of them $6 \%$ were treated with hypolipidaemic drugs. They smoked $5.9( \pm 10.6)$ cigarettes per day.
Apo C3 and Lp B:C3 concentrations (measured with the same electroimmunodiffusion method) were statistically significantly different between these subjects and our sample, as were BMI and hypolipidaemic treatment.

\section{Discussion}

In this cross-sectional study, we report an independent association between Lp B:C3 and macroangiopathy or coronaropathy in a group of patients with Type II diabetes. When submitted separately to multiple logistic regression analyses, triglycerides, apo C3 and Lp B:C3 were each independently associated with macrovascular complications but Lp B:C3 had the highest odds ratio. In addition we highlighted a dose-response relation between quartiles of Lp B:C3 and cardiovascular complications for $\mathrm{Lp} \mathrm{B}: \mathrm{C} 3$ of $10 \mathrm{mg} / \mathrm{l}$ or more.

The electroimmunodiffusion method used here to measure apo C3 and Lp B:C3 was first applied to the ECTIM cohort and showed an association between this variable and myocardial infarction. This method seems to be very sensitive and specific [9] and recognises apo C3 in Lp C3 non B and Lp B:C3 in an identical way.

In our study, subjects were hypertriglyceridaemic $(2.14 \pm 1.73 \mathrm{mmol} / \mathrm{l})$. The method used seems to be reliable in such subjects (as in Type II diabetic patients), as shown by plasma enrichment experiments with VLDL-TG, not exceeding $5.70 \mathrm{mmol} / \mathrm{l}$ (unpublished data). Only four of the subjects studied had higher triglyceride concentrations (Table 2).

It is likely that the glycation process could interfere with immunoassay analysis in diabetic patients. It appears, however, that apo $\mathrm{C} 3$ is not involved in these reactions because it has a very short half-life. Moreover we studied apo C3 and Lp B:C3 according to $\mathrm{HbA}_{1 \mathrm{c}}$ values ( $<$ or $>8.5 \%$ ): they were not statistically significantly different and were associated with macroangiopathy whatever the $\mathrm{HbA}_{1 \mathrm{c}}$ value. There is no published information on drug interference with aspirin or antiaggregant agents either in vivo or in vitro. Nevertheless according to a personal communication (G. Luc, Serlia, Lille, France) from the team who developed apo C3 and LpB:C3 measurement method, there is no interference with aspirin. Hypolipidaemic agents, particularly fibrates, do, however, interfere with apo C3 concentration because of their direct genomic effects through peroxisome proliferator-activated receptor (PPAR) $\alpha$ activation $[14,15]$. In our study, $19 \%$ of the patients were receiving hypolipidaemic treatment (and among them more than two-thirds were treated with fibrates). Even after exclusion of subjects treated with these drugs, the statistical association between triglycerides, apo C3, Lp B:C3 and macroan- 
giopathy or coronaropathy remained statistically significant.

Triglyceride, apo C3 and Lp B:C3 concentrations were statistically significantly lower in subjects receiving insulin treatment but their $\mathrm{HbA}_{1 \mathrm{c}}$ values did not differ from those of subjects treated by diet or with oral antidiabetic treatment. This suggests that insulin therapy influences lipoprotein metabolism independently from glycaemic control. Such results are of particular interest because apo C3 gene transcription is down-regulated by insulin. In some hypertriglyceridaemic families it has been shown moreover that there is a genetic variation (five single base pairs) in the promoter of the apo $\mathrm{C} 3$ gene in the "insulin response element" region, thus decreasing apo $\mathrm{C} 3$ gene transcription and creating an insulin-resistant state [16].

There is no information available on apo C3 or Lp $\mathrm{B}: \mathrm{C} 3$ in a diabetic population using this method. Statistically significantly higher apo C3 concentrations were shown in Type II diabetes with macrovascular complications in one study comparing lipid variables in two groups of 47 Type II diabetic patients with and without macrovascular disease. The concentrations of apo C3 were not comparable with those of our sample because the measurement methods for apo C3 were different (ELISA was used) [17]. In another study of concentration and composition of apo B-containing lipoproteins in Type II diabetes with ELISA an increased concentration of triglyceriderich Lp B:E and Lp B:C:E, of apo C3 and apo E independently from plasma triglycerides was shown [18]. Another study with ELISA in Type II diabetes observed that apo C3 and Lp B:C3 concentrations were increased [19].

Comparison of the values of apo C3 and Lp B:C3 between the French control group of the ECTIM study (men only) and our sample (men and women) showed significant differences ( $p<0.00001$ for each) but some variables were different, e. g. sex, BMI, hypolipidaemic drugs.

Several epidemiological studies on atherosclerosis $[20,21]$ have suggested that increased Lp B:C3 concentrations could be atherogenic, especially when linked to increased VLDL. The Lp B:C3 lipoprotein is among the triglyceride-rich lipoproteins with $\mathrm{Lp}$ $\mathrm{B}: \mathrm{E}$ and $\mathrm{Lp}$ B:C3:E associated with a "small dense LDL" phenotype defined using the classical centrifugation method which identifies heterogeneous lipoparticles. Both these closely associated phenotypes are known to be pro-atherogenic.

Diabetic dyslipidaemia is characterised by hypertriglyceridaemia and low HDL-cholesterol, and qualitative abnormalities such as preponderance of small, dense LDL [22], related to insulin resistance. Diabetic patients with normal triglyceride concentrations have an increased cardiovascular risk, probably linked to abnormal lipoprotein metabolism. There- fore in Type II diabetic patients with normal triglyceride concentrations VLDL are more highly accumulated in vitro in cultured macrophages than those of non-diabetic control subjects with normal triglyceride concentrations [23]. One of the determinants of this abnormal metabolism of lipoproteins could be apo C3. In subjects with normal triglyceride concentrations, apo $\mathrm{C} 3$ is mostly contained in fasting HDL and is transferred to VLDL in the hours following a meal. In subjects who are hypertriglyceridaemic, the majority of apo C3 is, however, found in VLDL during fasting [24]. There are no published findings on apo C3 metabolism in diabetic subjects, but it was recently shown in mixed hyperlipaemia (MHL) that even in the late postprandial state $(12 \mathrm{~h})$ apo $\mathrm{C} 3$ is mainly in LpB:C3 and that Lp B:C3-HDL remains low at that time [25] but this was not observed in hypercholesterolaemic subjects. Such variation was not observed in control subjects.

The main apolipoprotein of triglyceride-rich lipoproteins (TRLP), apo C3, plays an important part in TRLP metabolism and offsets the functions of apo $\mathrm{E}$ at each stage. The role of apo $\mathrm{C} 3$ has been deduced from animals and in vitro studies (diminished clearance of TRLP enriched with apo C3 [26], diminished interaction of triglycerides and apo C3-enriched lipoprotein B with hepatic LDL receptor [27, 28], hypertriglyceridaemia induced by overexpression of apo C3 in transgenic mice [29]) and confirmed in humans (it reduces TRLP catabolism [30], inhibiting their hepatic uptake by modifying the binding of apo $\mathrm{E}$ to LDL receptor $[31,32]$ and reducing lipolysis by inhibiting hepatic lipoprotein lipase [33]).

Apart from dyslipidaemia, regular cardiovascular risk factors also play a part in the incidence of macrovascular complications in patients with Type II diabetes [34]. In our multivariate analysis, there was an independent association between macroangiopathy and some well-known cardiovascular risk factors such as age, sex, duration of diabetes and microalbuminuria. The same associations were noted in patients with coronary disease with a weaker statistical significance due to a smaller number of patients.

As expected, subjects with retinopathy or microangiopathy had higher $\mathrm{HbA}_{1 \mathrm{c}}$ values than those without $(13.5 \pm 10.3 \%$ vs $8.5 \pm 8.2 \%, p<0.004$ for retinopathy), and a longer duration of diabetes (13.2 \pm 10.3 years vs $8.0 \pm 7.8$ years, $p<0.001)$.

In our sample of 188 patients with Type II diabetes, triglycerides, apo C3 and Lp B:C3 were independent markers of cardiovascular disease. Among those, Lp B:C3 was the most discriminating. There was a statistically significant dose-response relation between apo C3, Lp B:C3 and macroangiopathy or CHD (to a lesser extent in the latter due to the smaller size of the sample), with a statistically significantly higher prevalence of both when apo $\mathrm{C} 3$ concentration was $31 \mathrm{mg} / \mathrm{l}$ or more and Lp B:C3 was $10 \mathrm{mg} / \mathrm{l}$ or 
more. The apo C3 and Lp B:C3 values were lower in the insulin-treated group, though their glycaemic control evaluated by $\mathrm{HbA}_{1 \mathrm{c}}$ was similar. Among the lipid variables studied, only triglycerides, apo $\mathrm{C} 3$ and Lp B:C3 were independently associated with macrovascular complications, which emphasises the crucial role of TRLP in CHD in Type II diabetes.

\section{References}

1. Kannel WB, MacGee DL (1979) Diabetes and cardiovascular disease. The Framingham Study. JAMA 241: 2035-2038

2. Kannel WB (1985) Lipids, diabetes, and coronary heart disease: Insights from the Framingham study. Am Heart J 110: 1100-1107

3. Koskinen P, Mänttäri M, Manninen V, Huttunen J, Heinonen OP, Frick MH (1992) Coronary heart disease incidence in the Helsinki Heart Study. Diabetes Care 15: 820-825

4. Ginsberg HN (1996) Diabetic dyslipidemia:basis mechanisms underlying the common hypertriglyceridemia and low HDL cholesterol levels. Diabetes 45[Suppl 3]:S27-S31

5. Ginsberg HN (1991) Lipoprotein physiology in non diabetic and diabetic states:relation to atherogenesis. Diabetes Care 14: 839-855

6. Semenkovich CF, Heinecke JW (1997) The mystery of diabetes and atherosclerosis. Time for a new plot. Diabetes 46: 327-334

7. Fontbonne A, Eschwège E, Cambien F et al. (1989) Hypertriglyceridemia as a risk factor of coronary heart disease mortality in subjects with impaired glucose tolerance or diabetes. Results from the 11-year follow-up of the Paris Prospective Study. Diabetologia 32: 300-304

8. Puchois P, Alaupovic P, Fruchart JC (1985) Mise au point sur les classifications des lipoprotéines plasmatiques. Ann Biol Clin (Paris) 43: 831-840

9. Luc G, Fievet C, Arveiler D et al. (1996) Apolipoproteins $\mathrm{C}_{3}$ and $\mathrm{E}$ in apo B- and non apo B-containing lipoproteins in two populations at contrasting risk for myocardial infarction: the ECTIM study. J Lipid Res 37: 508-517

10. Parra HJ, Arveiler D, Evans AE et al. (1992) A case-controlled study of lipoproteins particles in two populations at contrasting risk for coronary heart disease: the ECTIM study. Arterioscler Thromb 12: 701-707

11. Rose G, MacCartney P, Reid DD (1977) Self administration of a questionnaire on chest pain and intermittent claudication. $\mathrm{Br}$ J Prev Soc Med 31: 42-48

12. Bennett PH, Haffner S, Kasiske BL et al. (1995) Screening and management of microalbuminuria in patients with diabetes mellitus: recommendations of the Scientific Advisory Board of the National Kidney Foundation from an ad hoc committee of the Council on Diabetes Mellitus of the National Kidney Foundation. Am J Kidney Dis 25: 107-12

13. Hosner DW, Lemeshow S (1989) Applied Logistic Regression. Wiley Series in Probability and Mathematical Statisics, John Wiley, New York

14. Auwerx J, Schoonjans K, Fruchart JC, Staels B (1996) Transcriptionnal control of triglyceride metabolism: fibrates and fatty acids change the expression of the LPL and apo $\mathrm{C}_{3}$ genes by activating the nuclear receptor PPAR. Atherosclerosis 124[Suppl]:S29-S37

15. Schoonjans K, Staels B, Auwerx J (1996) Role of the peroxysome proliferator-activated receptor (PPAR) in mediating the effects of fibrates and fatty acids on gene expression. J Lipid Res 37: 907-925

16. Li WW, Dammermann MM, Smith JD, Metzger S, Breslow JL, Leff T (1995) Common genetic variation in the promoter of the human apo $\mathrm{C}_{3}$ gene abolishes regulation by insulin and may contribute to hypertriglyceridemia. J Clin Invest 96: 2601-2605

17. Ikeda T, Ohtani I, Fujiyama K et al. (1991) Apolipoproteins levels in non insulin dependent diabetes mellitus with clinical macroangiopathy. Diabetes Metab 17: 373-378

18. Alaupovic P, Bard JM, Tavella M, Shafer D (1992) Identification of apo B-containing lipoprotein families in NIDDM. Diabetes 41[Suppl]:18-25

19. Patti L, Swimburn B, Riccardi G, Rivellese AA, Howard BV (1991) Alterations in very low density lipoprotein subfractions in normotriglyceridaemic type 2 diabetes. Atherosclerosis 91: $15-23$

20. Hodis HN, Mack WJ, Azen SP et al. (1994) Triglyceride- and cholesterol-rich lipoproteins have a differential effect on mild/ moderate and severe lesion progression as assessed by quantitative coronary angiography in a control trial of Lovastatin. Circulation 90: 42-49

21. Blankenhorn DH, Alaupovic P, Wickham E, Chin HP, Azen SP (1993) Prediction of angiographic change in native human coronary arteries and aortocoronary bypass grafts. Lipid and non lipid factors. Circulation 88: 2762-2770

22. Kreisberg RA (1998) Diabetic dyslipidemia. Am J Cardiol 82: 67U-73U

23. Kraemer FB, Chen Y-DI, Lopez RD, Reaven GM (1985) effects of type 2 diabetes mellitus on the uptake of very low density lipoproteins by thioglycolate elicited mouse peritoneal macrophages. J Clin Endocrinol Metab 61: 335-342

24. Fredenrich A (1998) Role of apolipoprotein C3 in triglyceride rich lipoproteins metabolism. Diabetes Metab 24: 490-495

25. Saïdi Y, Sich D, Camproux A, Egloff M, Federspiel MC et al. (1999) Interrelations between postprandial lipoprotein B/CIII particle changes and high-density lipoprotein subpopulation profiles in mixed hyperlipoproteinemia. Metabolism 48: 60-67

26. Aalto-Setälä K, Fisher EA, Chen X et al. (1992) Mechanism of hypertriglyceridemia in human apolipoprotein $\mathrm{C}_{3}$ transgenic mice. Diminished very low density lipoprotein fractional rate associated with increased apo $\mathrm{C}_{3}$ and reduced apo $\mathrm{E}$ on the particles. J Clin Invest 90: 1889-1900

27. Windler E, Chao Y, Havel RJ (1980) Regulation of the hepatic uptake of triglyceride-rich lipoproteins in the rat. Opposing effects of homologous apolipoproteins $\mathrm{E}$ and individual $\mathrm{C}$ apoproteins. J Biol Chem 255: 8303-8307

28. De Silva HV, Lauer SJ, Wang KH, Mahly RW, Taylor JM (1994) Overexpression of human apolipoprotein C III in transgenic mice. J Biol Chem 269: 2324-2335

29. Ito Y, Azrolan N, O’Connel A, Walsh A, Breslow JL (1990) Hypertriglyceridemia as a result of human apo $\mathrm{C}_{3}$ gene expression in transgenic mice. Science 249: 790-793

30. Ginsberg HN, Le Ns, Goldberg IJ et al. (1986) Apolipoprotein $\mathrm{B}$ metabolism in subjects with deficiency of apolipoprotein $\mathrm{C}_{3}$ and $\mathrm{A}_{1}$. Evidence that apolipoprotein $\mathrm{C}_{3}$ inhibits catabolism of triglyceride-rich lipoproteins by lipoprotein lipase in vivo. $\mathbf{J}$ Clin Invest 78: 1287-1295

31. Clavey V, Lestavel-Delattre S, Copin C, Bard JM, Fruchart JC (1995) Modulation of lipoprotein B binding to the LDL-receptor by exogenous lipids and apolipoproteins $\mathrm{C}_{1}, \mathrm{C}_{2}, \mathrm{C}_{3}$ and $\mathrm{E}$. Arterioscler Thromb Vasc Biol 15: 963-971

32. Clavey V, Agnani G, Bard JM, Lestavel-Delattre S, Fruchart JC (1991) Interaction entre le LDL-récepteur et les lipoprotéines contenant de l'apo B. Ann Endocrinol (Paris) 52: 459-463

33. Wang CS, MacConathy WJ, Kloer HU, Alaupovic P (1985) Modulation of lipoprotein lipase activity by apolipoproteins. Effect of apolipoprotein $\mathrm{C}_{3}$. J Clin Invest 75: 384-390

34. Wingard DL, Barrett-Connor E, Criqui MH, Suarez L (1983) Clustering of heart disease risk factors in diabetic compared to non diabetic adults. Am J Epidemiol 117: 19-26 